\title{
Efeito do substrato bovino na germinação de pimenta biquinho (capsicum chinense) irrigado com água residuária
}

\author{
Effect of bovine substrate on the germination of biquinho pepper (capsicum chinense) irrigated with wastewater \\ Viviane Farias Silva', Elka Costa Santos Nascimento², Leandro Oliveira Andrade ${ }^{3}$, \\ Jose Geraldo Vasconcelos Baracuhy4, Vera Lucia Antunes Lima ${ }^{4}$ \\ ' Mestranda em Irrigação e Drenagem, Universidade Federal de Campina Grande \\ ${ }^{2}$ Graduanda em Engenharia Agrícola, Universidade Federal de Campina Grande \\ ${ }^{3}$ Professor, Universidade Estadual da Paraiba, \\ ${ }^{4}$ Professores de engenharia agrícola, Universidade Federal de Campina Grande
}

\begin{abstract}
Resumo
O semiárido brasileiro caracterizado pela seca prolongada e chuvas más distribuídas, reduzindo a qualidade e quantidade de água disponível. Nesse contexto, a presente pesquisa realizada em ambiente protegido na Universidade Federal de Campina Grande objetivando-se analisar a germinação da pimenta biquinho com substrato bovino irrigado com diferentes níveis de água residuária tratada. Os tratamentos corresponderam a 5 níveis de água $(\mathrm{N})$ utilizando-se água de abastecimento e residuária proveniente do reator anaeróbico de fluxo ascendente (UASB) baseada na necessidade hídrica (NH) da cultura, sendo elas: $100 \%$ da NH (N5), $80 \%$ da NH (N4), $60 \%$ da NH (N3), 40 $\%$ da NH (N2) e $20 \%$ da NH (N1).Foram avaliados a porcentagem de germinação (PG), o índice de velocidade de germinação (IVG), a velocidade média $(\mathrm{Vm})$ e o tempo médio $(\mathrm{Tm})$ de germinação da pimenta biquinho ( BRS Moema). A porcentagem de germinação para a água de abastecimento teve média de $42,3 \%$ e 46,5 \% para residuária. Para os níveis de água aplicados a velocidade média de germinação da pimenta biquinho variou de 0,086 a 0,089. A germinação da pimenta teve melhores resultados com o uso da água residuária.O reuso de água em regiões semiáridas é uma alternativa viável reduzindo os custos e disponibilizando nutrientes a cultura.
\end{abstract}

Palavras-chaves: BRS Moema, Níveis de água, Substrato orgânico.

\begin{abstract}
The Brazilian semiarid region characterized by prolonged drought and poor rainfall distributed, reducing the quality and quantity of water available. In this context, the present study performed in a protected environment at the Federal University of Campina Grande aiming to analyze the germination of pepper pout with bovine substrate irrigated with different levels of wastewater treated. The treatments consisted on 5 water levels (N) using the water supply and wastewater derived from an upflow anaerobic (UASB) based on plant water requirement (NH), as follows: $100 \%$ of $\mathrm{NH}(\mathrm{N} 5)$ (PG) the germination speed index (GSI), mean velocity (MV) and the average time (Tm) of germination were evaluated, on biquinho pepper (BRS Moema). The germination percentage for water supply averaged $42.3 \%$ and $46.5 \%$ for wastewater. For water levels applied the average germination rate of biquinho pepper ranged from 0.086 to 0.089 . The germination of pepper had better results with the use of water residuária. $\mathrm{O}$ reuse of water in semi-arid regions is a viable alternative reducing costs and providing nutrients culture.
\end{abstract}

Keywords: BRS Moema, water levels, organic substrate. 


\section{INTRODUÇÃO}

A água é um recurso natural renovável importante para o desenvolvimento socioeconômico. O Brasil possui 13,8 \% da água doce do planeta, porém desigualmente distribuídas (MALVEZZI, 2012). A região semiárida é caracterizada pelas chuvas irregulares e secas prolongadas reduzindo a disponibilidade e usos da água, sendo um impasse na questão de seu desenvolvimento.

O tratamento de água residuária torna-se uma alternativa para o semiárido disponibilizando água para a agricultura reduzindo os impactos ambientais. De acordo com Asano et al., (2007) o reúso de água beneficia a preservação de fontes de qualidade elevada, proteção ambiental além de benefícios econômicos e sociais.

O uso de água residuária na irrigação pode reduzir os custos de fertilização das culturas, bem como o nível requerido de purificação do efluente e, consequentemente, os custos de seu tratamento, já que as águas residuárias contêm nutrientes e o solo e as culturas comportam-se como biofiltros naturais (BRANDÃO et al., 2002). A reutilização de água vinculada a uma boa estratégia de manejo na irrigação é fundamental para economizar água sem, no entanto, por em risco o rendimento das culturas (JALOTA et al., 2006 ; PEREIRA et al., 2009). Como a necessidade hídrica varia entre as espécies, e ao longo do seu ciclo, conhecer as respostas das espécies é de grande importância para a elaboração de planos de manejo adequados, de maneira a se obter rendimentos econômicos mais altos (MONTEIRO et al., 2006, LIMA et al., 2012).

$\mathrm{O}$ estudo de diferentes lâminas de irrigação constitui uma maneira bastante prática para se determinar as necessidades hídricas de uma espécie, em certa região para se estimar a quantidade de água que a cultura necessita para crescer e produzir dentro dos limites impostos por seu potencial genético (AZEVEDO \& BEZERRA, 2008). Por isso, as lâminas de irrigação tem sido objeto de estudo de vários pesquisadores nas mais diversas espécies, podendo-se mencionar Azevedo \& Bezerra (2008); Garcia et al., (2007) e Morais et al., (2008).

Para a comercialização de pimentas ornamentais, existem diversos fatores que interferem na produção, destacando-se a aparência da planta, tamanho do recipiente, substrato, controle sobre o crescimento, bem como necessidades hídricas e nutricionais, exigindo estudos sobre adubação, irrigação e fertirrigação (BARBOSA et al., 2011).

O cultivo de pimenta irrigado com reaproveitamento de água baseado na necessidade hídrica utilizando como substrato esterco orgânicos disponíveis em propriedades rurais constitui-se numa fonte de nutrientes economicamente viáveis reduzindo os custos com fertilizantes e água de qualidade. $\mathrm{Na}$ região do agreste Paraibano o esterco bovino é uma das principais fontes de adubação orgânica empregada pelos agricultores, pela disponibilidade local e baixo custo de aquisição, em alguns casos é a única utilizada para fertilização de culturas (GALVAO et al., 2008).

Nesse contexto, a pesquisa foi realizada objetivando-se analisar a germinação da pimenta biquinho com substrato bovino irrigada com diferentes níveis de água residuária tratada.

\section{MATERIAL E METODOS}

O experimento foi realizado em casa de vegetação, pertencente à Unidade Acadêmica de Engenharia Agrícola (UAEAg), da Universidade Federal de Campina Grande - UFCG, localizado no município de Campina Grande, Estado da Paraíba-PB, nas coordenadas geográficas $7^{\circ} 15^{\prime} 18^{\prime \prime}$ de latitude sul e 35'52'28" de longitude oeste, a uma altitude de 550 m (CPRM, 2005).

Utilizou-se 60 vasos plásticos $\mathrm{n}^{\circ} 17$, de cor preta, com as seguintes dimensões: $15 \mathrm{~cm}, 9 \mathrm{~cm}$ e 14 $\mathrm{cm}$, diâmetro superior, diâmetro inferior e altura respectivamente; e para a drenagem foram feitos 06 furos no fundo e colocado uma tela protetora em seguida foi preenchido com brita número 0 , cobrindo todo o fundo, na proporção 7: 3 de solo e substrato orgânico (bovino), 30 \% do volume total.

Os tratamentos corresponderam a 5 níveis de água $(\mathrm{N})$ utilizando-se água de abastecimento e residuária tratada por reator anaeróbio de fluxo ascendente (UASB) baseada na necessidade hídrica (NH) da cultura, sendo elas: $100 \%$ da NH (N5),80\% da NH (N4), $60 \%$ da NH (N3), $40 \%$ da NH (N2) e $20 \%$ da $\mathrm{NH}$ (N1). Cada unidade experimental foi formada de um recipiente contendo uma planta. O delineamento experimental adotado foi em blocos casualizados em esquema fatorial $(2 \times 5)$ com 3 repetições e duas plantas/ repetição, perfazendo 60 unidades experimentais. 
Foram avaliados diariamente até os 14 dias após a semeadura (DAS) a porcentagem de germinação (PG), o índice de velocidade de germinação (IVG), a velocidade média (Vm) e o tempo médio (Tm) de germinação da pimenta biquinho (BRS Moema). A porcentagem de germinação, o tempo médio de germinação e a velocidade média $(\mathrm{Vm})$ foram calculados de acordo com Labouriau \& Valadares (1976) e o índice de velocidade de emergência (IVG) foi determinado segundo Maguire (1962).

Os resultados foram avaliados por análise de variância e as médias comparadas pelo teste de Tukey a 5\% de significância com o auxilio do programa computacional Sistema para Análise de Variância - SISVAR (FERREIRA, 2003) para os dados obtidos nos diferentes tratamentos de natureza qualitativa, enquanto que para os dados de natureza quantitativa, form submetidos ao estudo de regressão linear e quadrática.

\section{RESULTADOS E DISCUSSÕES}

Analisando os resultados de acordo com a Tabela 1 o tempo médio e a velocidade média de germinação foram significativos estatisticamente em relação ao tipo de água ao nível de $5 \%$.

Tabela 01- Resumo da análise de variância para a porcentagem de germinação (PG), índice de velocidade de germinação (IVG), tempo médio (Tm) e velocidade média (Vm) germinação da pimenta biquinho.

\section{Quadrados Médios ${ }^{1}$}

\begin{tabular}{lccccc}
\hline Fonte de Variação & GL & $\mathbf{P G}^{2}$ & IVG $^{3}$ & Tm & Vm \\
Tipo de Água (A) & 1 & $0,65^{\mathrm{ns}}$ & $0,007^{\mathrm{ns}}$ & $1,14^{*}$ & $0,00007^{*}$ \\
Níveis de água (N) & 4 & $0,85^{\mathrm{ns}}$ & $0,025^{\mathrm{ns}}$ & $0,085^{\mathrm{ns}}$ & $0,000005^{\mathrm{ns}}$ \\
Regressão Linear & 1 & $0,26^{\mathrm{ns}}$ & $0,024^{\mathrm{ns}}$ & $0,024^{\mathrm{ns}}$ & $0,00002^{\mathrm{ns}}$ \\
Regressão Quadrática & 1 & $0,65^{\mathrm{ns}}$ & $0,013^{\mathrm{ns}}$ & $0,117^{\mathrm{ns}}$ & $0,000007^{\mathrm{ns}}$ \\
Desvio Regressão & 2 & $1,24^{\mathrm{ns}}$ & $0,032^{\mathrm{ns}}$ & $0,099^{\mathrm{ns}}$ & $0,000006^{\mathrm{ns}}$ \\
Interação (A * N ) & 4 & $0,51^{\mathrm{ns}}$ & $0,023^{\mathrm{ns}}$ & $0,045^{\mathrm{ns}}$ & $0,000002^{\mathrm{ns}}$ \\
Resíduo & 20 & 1,45 & 0,05 & 0,14 & 0,000008 \\
CV (\% ) & & 18,83 & 14,86 & 3,28 & 3,16 \\
& & & & & \\
\hline Aipo de Água & & & & & \\
\hline Água de abastecimento (A1) & & $42,3 \mathrm{a}$ & $1,74 \mathrm{a}$ & $11,45 \mathrm{a}$ & $0,087 \mathrm{a}$ \\
Água residuária (A2) & & $46,5 \mathrm{a}$ & $2,07 \mathrm{a}$ & $11,07 \mathrm{a}$ & $0,090 \mathrm{a}$
\end{tabular}

1 NS: não significativo $(\mathrm{P}>0,05)$; *: significativo $(\mathrm{P}<0,05)$; C.V.: coeficiente de variação. PG(\%); IVG(sementes/dia); Tm(dias); Vm(dias). Médias seguidas de mesma letra na vertical não diferem entre si pelo teste de Tukey.2 Transformação raiz quadrada- SQRT (Y) 3 Transformação Raiz quadrada de Y + 0.5 - SQRT ( Y + 0.5 ) 
Na Tabela 1 percebe-se que a porcentagem de germinação para a água de abastecimento teve média de $42,3 \%$ e 46,5\% para residuária com maior média. É provável que esse efeito positivo tenha ocorrido em função do caráter fertilizante que os efluentes domésticos possuem (BEZERRA \& FIDELES FILHO, 2009). Paiva et al., (2012) afirmam que a pimenta malagueta teve um melhor desempenho com o uso da água residuária. Segundo Marcos Filho (2005), para que a germinação ocorra, ela depende da presença de um nível ideal de hidratação dos tecidos, que possibilite a ativação dos processos metabólicos que culminam no desenvolvimento do eixo embrionário. Silva et al.,(2013) avaliando diferentes tipos de pimenta obtiveram após 30 dias da semeadura a porcentagem de germinação das variedades 'Dedo de Moça' (TECNOSEED), 'Tabasco' (TOP SEED), 'Vulcão 2011', e 'Chapéu de Bispo 2012', apresentavam, respectivamente, 34\%, 25\%, 5\% e 0\% de germinação. Enquanto que aos 14 dias após semeadura a pimenta biquinho em substrato bovino em diferentes níveis de água teve resultados superiores a 42,3\%. Conforme a Figura 1 para os níveis de água o N1(20\%) teve média superior com $50,18 \%$.

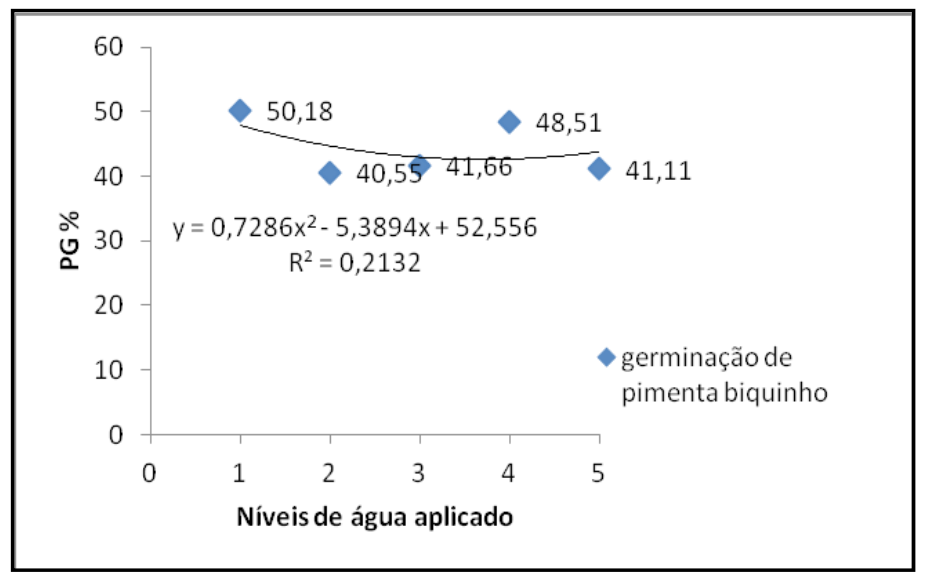

Figura 01- Porcentagem de germinação da pimenta biquinho irrigado com diferentes níveis de água residuária e de abastecimento.

Para o Índice de velocidade de germinação (IVG) de acordo com a Tabela 1 a água residuária teve maior média com 2,07 sementes/dia. Na Figura 2 o maior índice de velocidade de germinação com 2,14 sementes/dia para o nível de água aplicado a $20 \%$ da necessidade hídrica da planta N1

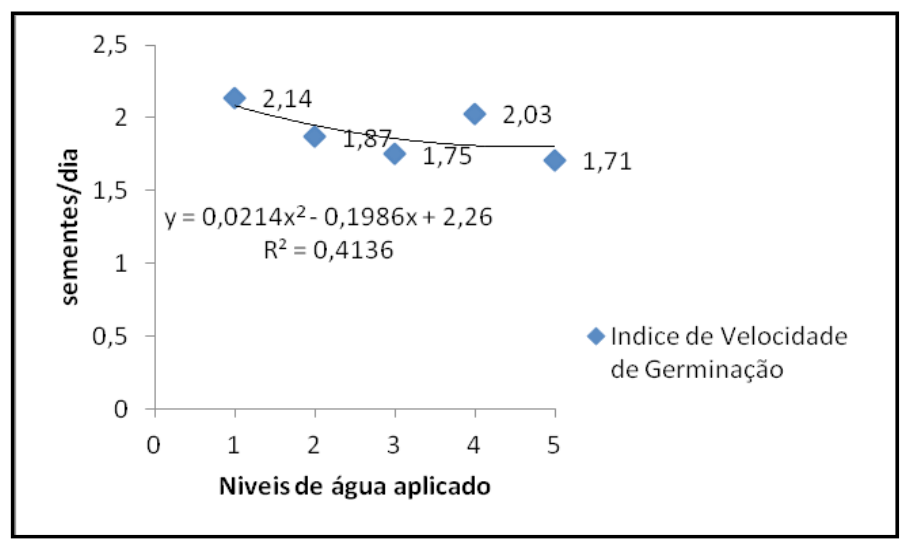

Figura 02- Índice de velocidade de germinação da pimenta biquinho irrigado com diferentes níveis de água residuária e de abastecimento.

Vieira \& Krzyzanowsky (1999) afirmaram que para variável IVG quanto maior o valor apresentado, maior é capacidade das sementes expressarem seu potencial germinativo. De acordo com Rabanni et al., (2013) a análise do tempo médio de germinação é importante para serem avaliadas como as condições do meio de instalação de um experimento podem influenciar na velocidade da embebição e, por conseguinte, o tempo médio de emergência da semente. O tempo médio de germinação para 
água de abastecimento foi de 11,45 (Tabela 1).Na Figura 3 o tempo médio de germinação em relação aos níveis de água aplicado variou de 11,08,a 11,42, para N1 (2\%) e N2(40\%), respectivamente.

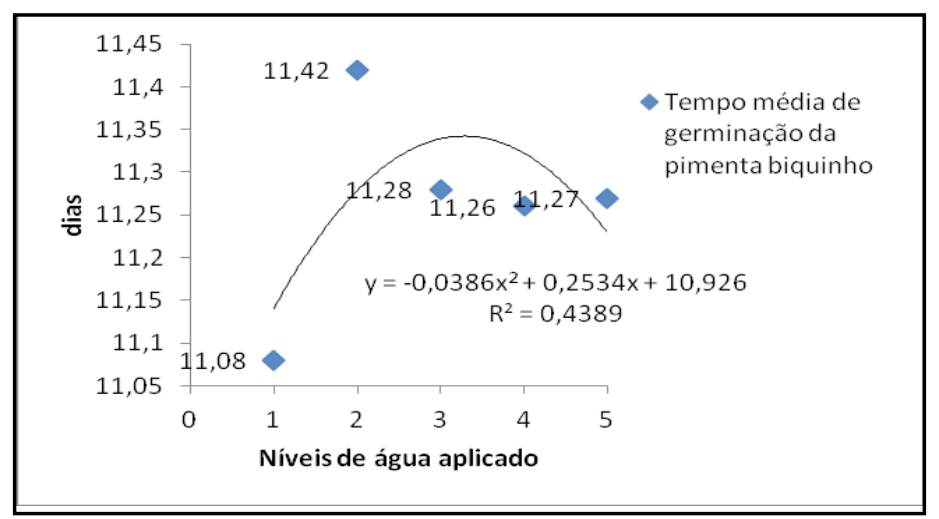

Figura 03- Tempo médio de germinação da pimenta biquinho irrigado com diferentes níveis de água residuária e de abastecimento.

A velocidade média de germinação para a água residuária (Tabela 1) foi de 0,09 e para água de abastecimento 0,087 havendo estatisticamente diferença significativa ao nível de $5 \%$. Para os níveis de água aplicados a velocidade média de germinação da pimenta biquinho variou de 0,086 a 0,089 de acordo com a Figura 4.

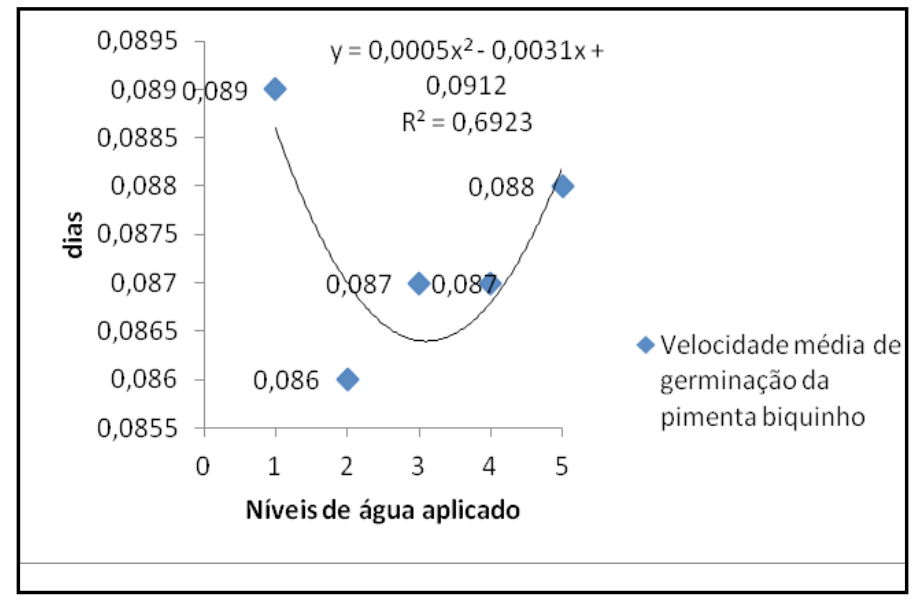

Figura 04- Velocidade média da germinação da pimenta biquinho irrigada com diferentes níveis de água residuária e de abastecimento.

Segundo Calbo et al. (2009), uma redução da velocidade do processo de germinação ou emergência indica que houve um declínio fisiológico da semente, mostrando, portanto, que fatores extrínsecos como as condições ambientais exigentes pela cultura são decisivas nesse processo.

\section{CONCLUSÃO}

O tipo de água aplicada influênciou no tempo e velocidade de germinação da pimenta biquinho.

O uso de água residuária em áreas semiáridas é uma alternativa para a irrigação na agricultura.

O substrato orgânico de solo/esterco bovino torna viável a produção de mudas ornamentais, reduzindo os custos. 


\section{REFERÊNCIAS}

ASANO, T.; BURTON, F.; LEVERENZ, H.; TSUCHIHASHI, R.; TCHOBANOGLOUS, G.. Water reuse, issues, technologies, and applications. New York: Metcalf \& Eddy/AECOM; McGraw Hill, 2007.

AZEVEDO, J. H. O. de; BEZERRA, F. M. L. Resposta de dois cultivares de bananeira a diferentes lâminas de irrigação. Revista Ciência Agronômica, v. 39, n. 01, p. 28 - 33, 2008.

BARBOSA, J. G.; MUNIZ, M. A.; MESQUITA, D. Z.; COTA, F. O. BARBOSA, J. M.; MAPELI, A. M.; PINTO, C. M. F.; FINGER, F. L. Doses de solução nutritiva para fertirrigação de pimentas ornamentais cultivadas em vasos.Revista Brasileira de Horticultura Ornamental. V. 17, Nº.1, 2011, p. 29-36.

BEZERRA, B. G.; FIDELES FILHO, J. Análise de crescimento da cultura do algodoeiro irrigada com águas residuárias.Revista Ciência Agronômica, v.40, p.339-345, 2009.

BRANDAO, L. P.; MOTA, S.; MAIA, L. F.. Perspectivas do Uso de Efluentes de Lagoas de Estabilização em Irrigação. In: VI Simpósio Ítalo Brasileiro de Engenharia Sanitária e Ambiental, 2002, Vitória, ES. Anais do VI SIBESA. Rio de Janeiro: ABES, 2002.

BRASIL. Ministério das Minas e Energia. Secretaria de Minas e Metalurgia; CPRM - Serviço Geológico do Brasil. Projeto cadastrado de fontes de abastecimento por água subterrânea(Diagnostico do município de Campina Grande-Paraiba). Brasília: CPRM, 2005. Disponível em $:<$ http://www.cprm.gov.br/rehi/atlas/ paraiba/relatorios/CAMP050.pdf> Acesso em 15/07/2014

CALBO, A. G.; AROCA, S. C. Medidas para mitigar os efeitos das mudanças climáticas na produção de hortaliças. In: Guedes, M. R. (Ed.). Mudanças climáticas globais e a produção de hortaliças. Brasília, DF: Embrapa Hortaliças, 2009. p. 95-126

FERREIRA, D. F. SISVAR. Versão 4.3. Lavras: UFLA, 2003. Software.

GALVAO, S. R.; SALCEDO, I. H.; OLIVEIRA, F. F. Acumulacao de nutrientes em solos arenosos adubados com esterco bovino. Pesquisa Agropecuária Brasileira, v. 43, n. 01, p. 99-105, 2008.

GARCIA, F. C. de H.; BEZERRA, F. M. L; FREITAS, C. A. S. de. Níveis de irrigação no comportamento produtivo do mamoeiro Formosa na Chapada do Apodi, CE. Revista Ciência Agronômica, v. 38, n. 02, p. 136-141, 2007.

MORAIS, N. B.; BEZERRA, F. M. L.;MEDEIROS, J. F.; CHAVES, S. W. P. Resposta de plantas de melancia cultivadas sob diferentes níveis de água e de nitrogênio. Revista Ciência Agronômica, v. 39, n. 03, p.369-377, 2008.

JALOTA, S. K.; SOOD, A. G. B. S.; CHAHAL, B. U. Crop water productivity of cotton ( Gossypium hirsutum L.)- wheat (Triticum aestivum L.) system as influenced by déficit irrigation, soil texture and precipitacion. Agricultural Water Management, v. 84, p. 137-16,2006.

LABORIAU, L. G. \& VALADARES, M. B. (1976). On the germination of seeds of Calotropis procera. Anais da Academia Brasileira de Ciências, São Paulo, 48:174-186.

LIMA, M. E.; CARVALHO, D. F.; SOUZA, A. P.; ROCHA, H. S.; GUERRA, J. G.; Desempenho do cultivo da berinjela em plantio direto submetida a diferentes lâminas de irrigação. Revista Brasileira de Engenharia Agrícola e Ambiental, Campina Grande, v. 16, n. 6, p. 604-610, 2012

MAGUIRE, J.D. (1962). Speed of germination aid in selection and evaluation for seedling emergence and vigor. Crop Science, Madison, 2(2):176-177. 
MALVEZZI, R. Semi-arido - uma visao holistica. - Brasilia: Confea, 2007. 140p. - (Pensar Brasil)

MARCOS FILHO, J. Fisiologia de sementes de plantas cultivadas. Piracicaba: FEALQ, 2005. 495p.

MONTEIRO, R. O. C.; COLARES, D. S.; COSTA, R. N. T.; LEÃO, M. C. S.; AGUIAR, J. V. Função de resposta do meloeiro a diferentes lâminas de irrigação e doses de nitrogênio. Horticultura Brasileira, Brasília, DF, v. 24, n. 4, p. 455-459, 2006.

PAIVA, L.A.L; ALVES, S.M.C; BATISTA, R.O; OLIVEIRA, J.F; COSTA, M.S; COSTA, J.D. Influência da aplicação de esgoto doméstico terciário na produção de mudas de pimenta malagueta. In: Inovagri International Meeting e VI Winotec, 2012. Anais... Fortaleza, 2012. 6p.

PEREIRA, L. S.; PAREDES, P.; EHOLPANKULOV, E. D.; INCHENKOVA, O. P.; TEODORO, P. R.; HORST, M. G. Irrigation scheduling strategies for cotton to cope with water scarcity in the Fergana Valley, Central Asia. Agricultural Water Management, v. 96, p.723-735,2009.

RABBANI, A. C. R.; SILVA-MANN, R.; FERREIRA, R. A.; CARVALHO, S. V. A.; NUNES, F. B. S.; BRITO, A. S. Efeito do estresse salino sobre atributos da germinação de sementes de girassol. Scientia Plena, Vol. 9, Num. 5, 2013.

SILVA, B. R.; SCHARDOSIM, S. E.; SELAU, D. E.; CANDIA, A. S. F.; SEIBERT, E. Avaliação da germinação e do desenvolvimento das mudas de diferentes variedades de pimentas. $2^{\circ}$ Simpósio de Integração Científica e Tecnológica do Sul Catarinense - SICT-Sul, 2013.

VIEIRA, R. D.; KRZYZANOWSKI, F. C. Teste de condutividade elétrica. In: Krzyzanowski, F.C.; Vieira, R.D.; França Neto, J.B. (Ed.). Vigor de sementes: conceitos e testes. Brasília: Abrates, 1999. Cap. 4, p. 1-26. 